\title{
Lusioersily
}

\section{MALDI Matrix Application Utilizing a Modified 3D Printer for Accessible High Resolution Mass Spectrometry Imaging}

Cobice, D., Mackay, L., Tucker, L., Conde-González, A., Campbell, C., Clarke, D., Richard, G., \& Hamm, G. (2018). MALDI Matrix Application Utilizing a Modified 3D Printer for Accessible High Resolution Mass Spectrometry Imaging. Analytical Chemistry, 90(15), 8742-8749. https://doi.org/10.1021/acs.analchem.8b00670

Link to publication record in Ulster University Research Portal

\section{Published in:}

Analytical Chemistry

Publication Status:

Published (in print/issue): 04/06/2018

DOI:

10.1021/acs.analchem.8b00670

\section{Document Version}

Author Accepted version

\section{General rights}

Copyright for the publications made accessible via Ulster University's Research Portal is retained by the author(s) and / or other copyright owners and it is a condition of accessing these publications that users recognise and abide by the legal requirements associated with these rights.

\section{Take down policy}

The Research Portal is Ulster University's institutional repository that provides access to Ulster's research outputs. Every effort has been made to ensure that content in the Research Portal does not infringe any person's rights, or applicable UK laws. If you discover content in the Research Portal that you believe breaches copyright or violates any law, please contact pure-support@ulster.ac.uk. 


\section{analytical chemistry}

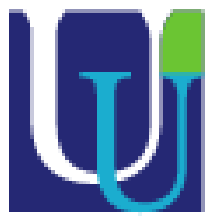

\section{Technical Note}

\section{MALDI Matrix Application Utilizing a Modified 3D Printer for Accessible High Resolution Mass Spectrometry Imaging}

Lulu Tucker, Antonio Conde-González, Diego Cobice, Gregory Hamm, Richard J. A. Goodwin, Colin J. Campbell, David James Clarke, and Colin Logan Mackay

Anal. Chem., Just Accepted Manuscript • DOI: 10.1021/acs.analchem.8b00670 • Publication Date (Web): 04 Jun 2018

Downloaded from http://pubs.acs.org on June 6, 2018

\section{Just Accepted}

"Just Accepted" manuscripts have been peer-reviewed and accepted for publication. They are posted online prior to technical editing, formatting for publication and author proofing. The American Chemical Society provides "Just Accepted" as a service to the research community to expedite the dissemination of scientific material as soon as possible after acceptance. "Just Accepted" manuscripts appear in full in PDF format accompanied by an HTML abstract. "Just Accepted" manuscripts have been fully peer reviewed, but should not be considered the official version of record. They are citable by the Digital Object Identifier (DOI®). "Just Accepted" is an optional service offered to authors. Therefore, the "Just Accepted" Web site may not include all articles that will be published in the journal. After a manuscript is technically edited and formatted, it will be removed from the "Just Accepted" Web site and published as an ASAP article. Note that technical editing may introduce minor changes to the manuscript text and/or graphics which could affect content, and all legal disclaimers and ethical guidelines that apply to the journal pertain. ACS cannot be held responsible for errors or consequences arising from the use of information contained in these "Just Accepted" manuscripts. 


\title{
MALDI Matrix Application Utilizing a Modified 3D Printer for Ac- cessible High Resolution Mass Spectrometry Imaging
}

\author{
Lulu H. Tucker ${ }^{1}$ Antonio Conde-González ${ }^{1}$ Diego Cobice ${ }^{2}$ Gregory R. Hamm ${ }^{3}$ Richard J. A. Goodwin ${ }^{3}$ \\ Colin J. Campbell ${ }^{1}$ David J. Clarke ${ }^{1}$ C. Logan Mackay* ${ }^{1}$ \\ ${ }^{1}$ EaStCHEM School of Chemistry, University of Edinburgh, UK EH9 3FJ \\ ${ }^{2}$ School of Biomedical Sciences, University of Ulster, UK BT52 1SA \\ ${ }^{3}$ Pathology Sciences, Drug Safety \& Metabolism IMED Biotech Unit, AstraZeneca, Cambridge Science Park, Milton Road, \\ Cambridge, UK CB4 0WG
}

Mass Spectrometry Imaging, Matrix-assisted laser desorption ionization, spheroids, 3D cell culture

\begin{abstract}
Successful matrix-assisted laser desorption ionization (MALDI) mass spectrometry imaging (MSI) relies on the selection of the most appropriate matrix and optimization of the matrix application parameters. In order to achieve reproducible high spatial-resolution imaging data, several commercially available automated matrix application platforms have become available. However, the high cost of these commercial matrix sprayers is restricting access into this emerging research field. Here, we report an automated platform for matrix deposition, employing a converted commercially-available 3D printer (\$300) and other parts commonly found in an analytical chemistry lab alow-cost alternative to commercial sprayers. Using printed fluorescent rhodamine B microarrays and employing experimental design, the matrix deposition parameters were optimized to minimize surface analyte diffusion. Finally, the optimized matrix application method was applied to image 3-dimensional MCF-7 cell culture spheroid sections (ca. $500 \mu \mathrm{m}$ diameter tissue samples) and sections of mouse brain. Using this system, we demonstrate robust and reproducible observations of endogenous metabolite and steroid distributions with a high spatial resolution.
\end{abstract}

\section{Introduction}

Over the last decade, MALDI mass spectrometry imaging (MSI) has become established as a powerful technique for observing the spatial distribution of small molecules, ${ }^{1}$ lipids, ${ }^{2}$ peptides $^{3}$ and proteins ${ }^{4}$ from the surface of a wide variety of samples. Using this technique, a spatial resolution of below 10 $\mu \mathrm{m}$ has been demonstrated. ${ }^{5-8}$ However, in order to achieve such robust high spatial resolution data, reproducible sample preparation is crucial. Each stage in the preparation workflow must be carefully optimized for the specific tissue or sample to maintain the true spatial distributions of the biomolecules and reduce inter-sample variability. ${ }^{9,10}$ During matrix application, analyte diffusion is a major concern, and the selection of the most appropriate matrix and optimization of its application parameters is key to obtaining high quality and biologically relevant spectra directly from tissue samples. ${ }^{11}$ Subtle changes in application rate, drying times, matrix composition and area density all affect the ionization efficiency, the propensity to detect low abundance compounds and the reproducibility of the results obtained. ${ }^{9}$ The matrix crystal dimensions produced also affect the lateral resolution of the image, thus optimization of matrix application can produce a crystal size smaller than the diameter of the laser, making the laser beam the limiting factor in the imaging resolution that can be achieved. ${ }^{9,11}$

Matrix can be applied with or without the addition of solvents. Solvent-free application can reduce analyte delocaliza- tion, whilst solvents can increase the number of peaks observed, producing complementary biological information. ${ }^{12}$ Solvent-based matrices can be applied either manually or using an automated robotic system, discretely as spots or continuously as a coating. ${ }^{11,13}$ During manual matrix application, the homogeneity of the matrix coverage and the crystal size often varies depending on the operator whilst automated methods have been developed with the goal of removing inter-user variation. ${ }^{14}$ Automated sprayers remove the variability by controlling the temperature, solvent flow rate, spray velocity and the number of passes; however, they are typically slower than the manual methods. ${ }^{12}$ A number of automated systems are commercially available, including the Bruker ImagePrep (Bremen, Germany), the SunChrom SunCollect (Friedrichsdorf, Germany) and the HTX-TM-Sprayer (HTX Technologies, LLC, NC, USA), however the cost of this equipment limits many research facilities' accessibility to the technique. ${ }^{15}$ Several less expensive automated matrix application strategies have been developed in academic research laboratories. These include, an automated acoustic spotter, ${ }^{13}$ the repurposing of an inkjet printer, ${ }^{16}$ and the use of an pneumatic sprayer on top of a pneumatically moveable base. ${ }^{15}$ These alternative printing nozzles apply different technologies to emit a spray of matrix capable of producing small uniform crystals suitable for MSI. Continuous inkjet (CIJ) printing is a low-cost method that pumps fluid through a nozzle to eject a continuous stream of uniform droplets at high frequency. ${ }^{17}$ The vibrations of a pie- 
zoelectric actuator determines the size of the droplets, generating droplets around $40 \mu \mathrm{m}$ in diameter, producing low resolution printing. ${ }^{17}$ To reduce the droplet size produced and increase the resolution, a number of drop-on-demand (DOD) printers have become commercially available, utilizing piezoelectric printing. ${ }^{18}$ This involves applying a voltage to alter the pulse duration and the diameter of the nozzle which then controls the size of the droplets. ${ }^{17} \mathrm{~A}$ disadvantage of this method is the high cost of the printer equipment required, as well as inkjet printers being prone to blockage of the nozzle. Alternatively, in the absence of a current, a pneumatic nebulizer works using induction to atomize the liquid using a gas stream and produces droplets of down to $0.1 \mu \mathrm{m} .{ }^{19}$ This method requires a lower flow rate than the inkjet options, thus using less liquid. $^{20}$

In this paper, we report an automated method for matrix deposition, employing a converted commercial 3D printer $(\$ 300)$ and other parts commonly found in an analytical chemistry lab. Using printed rhodamine B microarrays and fluorescence imaging, we quantitate the extent of diffusion during matrix application. Through the use of experimental design, key experimental parameters, including temperature, gas pressure and nozzle height were varied and the effect on analyte diffusion established. The optimization can be readily adapted for a range of similar devices. After optimization, matrix deposition using this system effectively reduced analyte diffusion. To demonstrate the use of this equipment, the optimized matrix application method was applied to observe metabolite distributions in MCF-7 spheroid sections (ca. $500 \mu \mathrm{m}$ diameter tissue samples) with a consistently high spatial resolution. The reproducibility and efficacy of the matrix application platform across larger samples was shown using mouse brain tissue sections (ca. $20 \mathrm{~mm}$ diameter) and with alternative matrices.

\section{Experimental}

Adaptation of a commercial 3D printer to a MALDI matrix application platform and optimization of matrix application conditions. An i3 duplicator 3D printer (WANHAO, Zhejiang, China) with a temperature controlled build plate (0$120^{\circ} \mathrm{C}$ ) was used in this work. Adaptation of the printer takes half an hour, with all equipment costing a total of ca. \$2600. See supplementary information for step-by-step instructions. Matrix deposition was achieved by replacing the printing extruder with a commercially available electrospray emitter for nebulization (Bruker Daltonics, Bremen, Germany). While comparatively expensive, a commercially available nebulizer was chosen as it is a robust device whose results can be replicated in other laboratories; and it will be commonly available in mass spectrometry laboratories. In these experiments no electrospray voltage was applied to the nebulizer relative to the sample slide. The $x, y, z$ position and feed rate of the extruder is controlled with G-code instructions; a numerical control programme developed by Massachusetts Institute of Technology. The $x, y$ positions were determined to cover the area of a slide, whilst the $z$ height was determined as a distance between the emitter and the bed height. The $z$ height was calibrated to 0 when the nebulizer was $2 \mathrm{~mm}$ above the bed.
To convert the 3D printer, the printer's extruder was removed and replaced with the nebulizer (Bruker Daltonics, Bremen, Germany) mounted onto a spacer fabricated from poly lactic acid (PLA) plastic and produced by 3D printing (figure 1). The $3 \mathrm{D}$ printed spacer was designed using an online software and converted to a .STL file for printing (see supplementary information for details).

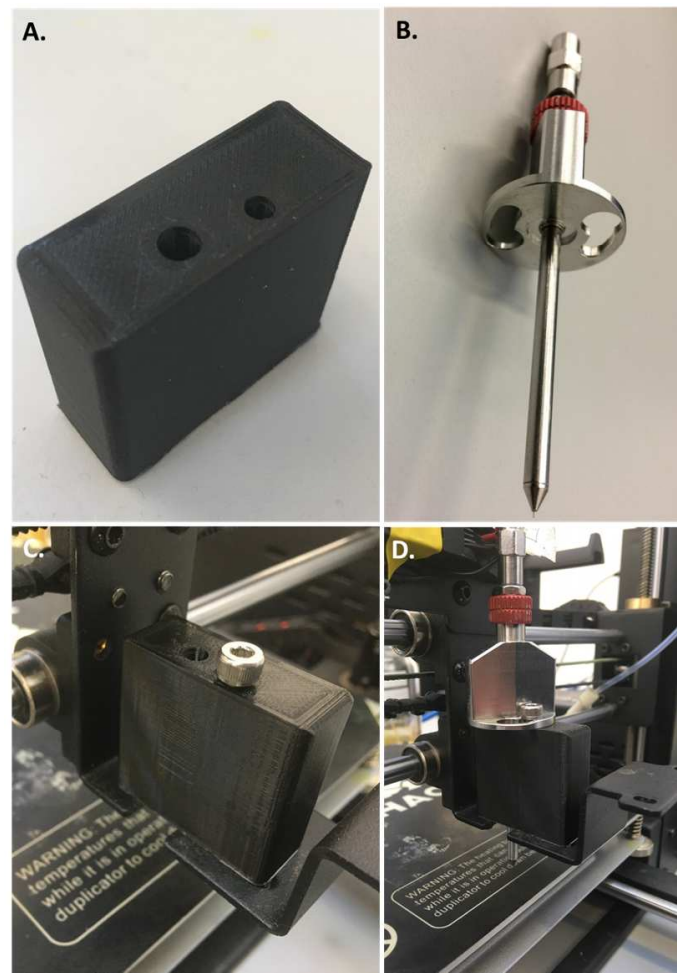

Figure 1. The printer extruder was removed and replaced with a (A) 3D printed block that held the (B) nebulizer in place. First, the (C) 3D block was screwed onto the structure and (D) subsequently the nebulizer held in place with a screw.

Nitrogen was used as the nebulizer gas and set manually with an inline regulator. The nebulizer was connected to a nitrogen supply (70 psi) and the inlet of the sprayer was connected to a Rheodyne C1-2006 6 port valve (Valco Instruments, Texas, USA) using $100 \mu \mathrm{m}$ internal diameter fused silica tubing (supplementary figure 1). The valve was equipped with a $5 \mathrm{~mL}$ sample loop, through which matrix could be injected and subsequently sprayed onto the slide. A Shimadzu LC-10ad HPLC pump (Shimadzu, Milton Keynes, UK) was connected to the 6 port valve at a flow rate of $0.1 \mathrm{~mL} /$ minute (figure 2 ). The matrix solution and application solvent used was 70:30 (v/v) acetonitrile: water. The mass of matrix deposited by the optimized method on the commercial TM-sprayer ${ }^{\mathrm{TM}}$ (HTX Technologies, LLC, NC, USA) was $2 \mathrm{mg}$. Therefore, the feed rate of the spray head and the flow rate of the solvent were optimized to match this area density. The velocity was set within the Gcode as F1000, which resulted in a velocity of 1100 $\mathrm{mm} /$ minute. The total time taken for 8 passes of a glass slide was 25 minutes. For consistent performance, regular wash cycles were done with solvent before and after use. 

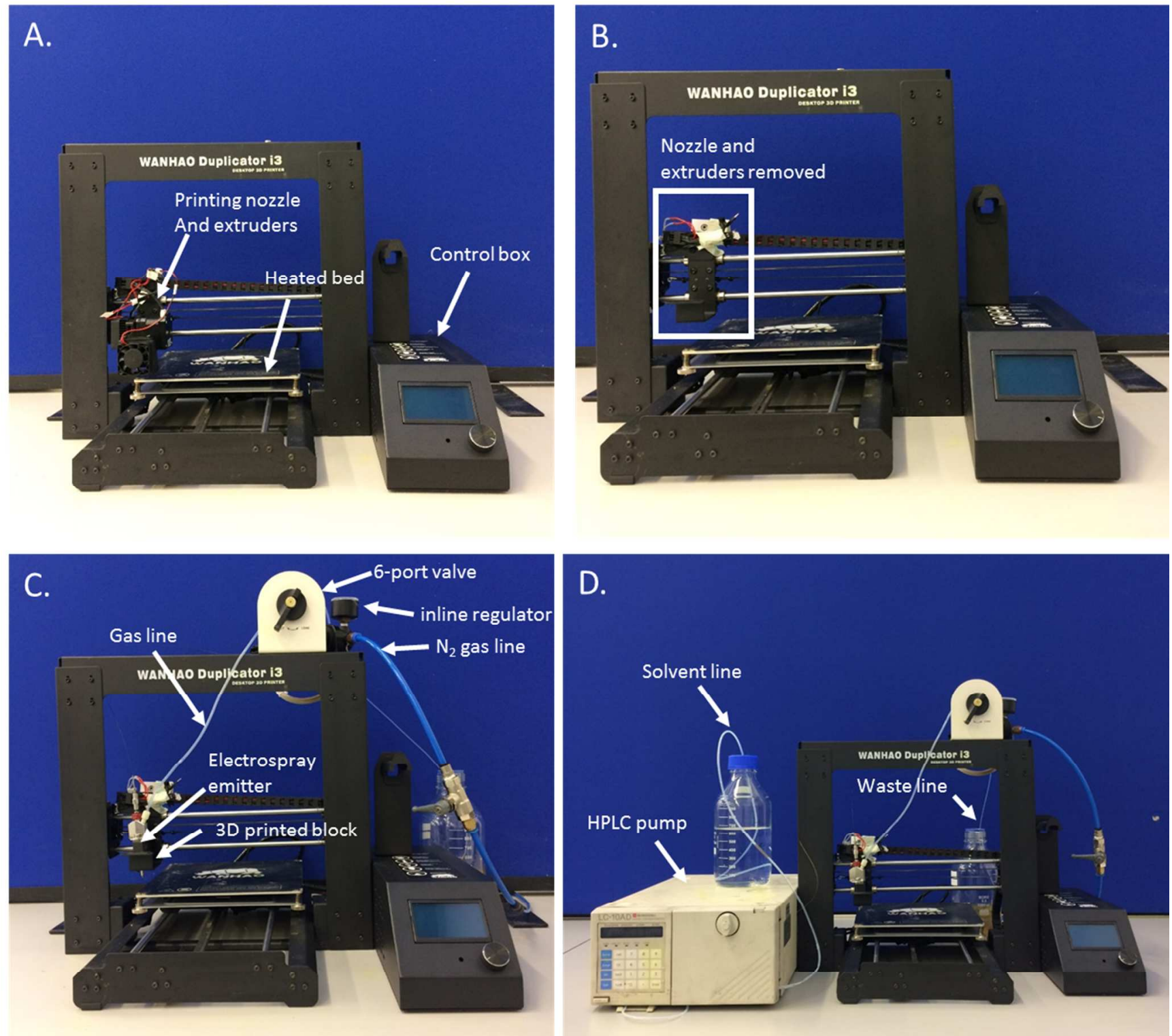

Figure 2. Outline of the printer setup. (A) The 3D printer used was the WANHAO Duplicator i3. (B) The extruder and fans were removed to leave the extruder housing block and remaining wires were insulated and secured in place with electrical tape. (C) A 3D printed block and nebulizer were secured in place and connected to a gas line and a 6-port HPLC valve with a $5 \mathrm{~mL}$ loop and a waste line. (D) An HPLC pump was connected and a solution of 70:30 (v/v) acetonitrile: water and a flow rate of $0.1 \mathrm{~mL} / \mathrm{minute}$ was used during matrix application.

Generation and matrix coating and fluorescence imaging of rhodamine $B$ microarrays. 45 spots containing 50 droplets of $350 \mathrm{pL} 0.01 \%$ rhodamine B (w/v) in water (Sigma-Aldrich, Missouri, USA) were dispensed onto a SuperFrost ${ }^{\mathrm{TM}}$ glass slide using a SciFLEXARRAYER S5 microarray printer (Scienion AG, Berlin, Germany) equipped with a PDC 80 dispense capillary (Piezo Systems, Massachusetts, USA) with a $50 \mu \mathrm{m}$ nozzle aperture. HPLC-grade water used as the SciFLEXARRAYER solvent and was degassed preceding printing by sonication (30 minutes). The fluorescence was measured using a BioAnalyzer 4F/4S (LaVision BioTec, Bielefeld, Germany) fluorescence scanner at $70 \mathrm{~ms}$ exposure time using a Cy5 filter. The area of observed fluorescence was measured using the circle area selection tool on ImageJ before and after matrix application. The standard deviation across the 45 spots was calculated before and after matrix application. 5 $\mathrm{mg} / \mathrm{mL}$ 9-aminoacridine (9AA) in 70:30 (v/v) acetonitrile: water was used as the matrix with the exact parameters outlined in the discussion. A flow rate of $0.1 \mathrm{~mL} /$ minute and nozzle velocity of $1100 \mathrm{~mm} /$ minute was used for both the printer and commercial TM-sprayer ${ }^{\mathrm{TM}}$.

Scanning electron microscopy. The matrix-coated rhodamine B slides were mounted on aluminium stubs with carbon tabs attached and sputter coated with $20 \mathrm{~nm}$ gold palladium (Emscope, Island Scientific, Ventnor, UK). The samples were viewed using a Hitachi S-4700 scanning electron microscope (Tokyo, Japan).

Cell culture and spheroids preparation. MCF-7 cells were donated at passage 52 from the Queen's Medical Research Institute (QMRI), Edinburgh (UK). Both monolayer cells and spheroids were grown in Dulbecco's Modified Eagles Medium 
(DMEM) (Thermo-Fisher, Massachusetts, USA) supplemented with $10 \%$ fetal calf serum (FCS) (Sigma Aldrich, Missouri, USA) and $1 \%$ penicillin (Thermo-Fisher, Massachusetts, USA). Monolayer cells were routinely seeded every 4 days in $75 \mathrm{~cm}^{3}$ flasks with $1 \mathrm{~mL}$ cells and $9 \mathrm{~mL}$ fresh media. The media was replaced every 48 hours.

Spheroids were obtained using the hanging drop method. ${ }^{21}$ Approximately 6000 cells were seeded into $20 \mu \mathrm{L}$ droplets that were then suspended from the lid of a petri dish. A reservoir of $10 \mathrm{~mL}$ media was placed in the bottom of the dish. Spheroids were fed with $5 \mu \mathrm{L}$ fresh media every 48 hours. Spheroids were incubated at $36.5{ }^{\circ} \mathrm{C}, 5 \% \mathrm{CO}_{2}$ and $95 \%$ humidity for 8 days before imaging.

\section{MALDI imaging sample preparation}

Sample preparation of spheroids. 8 day old untreated MCF7 spheroids were prepared using the following method. Media was removed and spheroids were washed with ammonium formate $(20 \mu \mathrm{L}, 50 \mathrm{mM})$. Each spheroid was picked up and placed into a droplet from which maximal liquid was removed. Blue dyed gelatin $(40 \mu \mathrm{L}, 10 \% \mathrm{w} / \mathrm{v})$ was placed onto the spheroids and frozen on isopentane and dry ice (2 minutes). The gelatin blocks were then snap frozen in liquid nitrogen and stored $\left(-80{ }^{\circ} \mathrm{C}\right)$ until sectioning. Sectioning was performed using a cryostat $\left(-21^{\circ} \mathrm{C}\right)$ (Leica CM 1900 (Leica Biosystems, Nussloch, Germany). Sections $(15 \mu \mathrm{m})$ were cut and thaw mounted onto conductive indium tin-oxide (ITO)-coated glass slides (Bruker Daltonics, Bremen, Germany). Consecutive sections were taken for haematoxylin and eosin staining.

Sample preparation of mouse brain: Licensed procedures were performed under the UK Animals (Scientific Procedures) Act, 1986. C57BL/6 mice (6-7 weeks, male) were from Harlan Olac Ltd. (Bicester, UK). Animals were killed by decapitation at 09:00 h. Brain tissue was snap frozen in liquid nitrogen and stored $\left(-80^{\circ} \mathrm{C}\right)$ until MSI analysis. Tissue sectioning was adapted from Cobice et al. ${ }^{22}$ Briefly, sectioning was performed using a cryostat (Leica Microsystems Inc., Bannockburn, IL, USA) and gelatin solution ( $10 \% \mathrm{w} / \mathrm{v})$ as embedding media. Sagittal brain sections $(10 \mu \mathrm{m})$ were cut and thaw mounted onto conductive ITO-coated glass slides (Bruker Daltonics, Bremen, GmbH) pre-coated with GirT-reagent $\left(0.15 \mathrm{mg} / \mathrm{cm}^{2}\right)$. Tissue sections were stored in a vacuum desiccator (RT, 1h) and then at $-80^{\circ} \mathrm{C}$ until MSI analysis.

Matrix application of MCF-7 spheroids. The matrix solution was $5 \mathrm{mg} / \mathrm{mL}$ of $9 A A$ in $70: 30(\mathrm{v} / \mathrm{v})$ acetonitrile: water. For the commercial sprayer, the TM-sprayer ${ }^{\mathrm{TM}}$ (HTX Technologies, LLC, NC, USA) was used to apply 8 coats of matrix at a flow rate of $0.1 \mathrm{~mL} /$ minute at $80{ }^{\circ} \mathrm{C}$ with a gas pressure of 10 psi and a velocity of $1100 \mathrm{~mm} /$ minute. Matrix area density was $0.11 \mathrm{mg} / \mathrm{cm}^{2}$. The optimized converted 3D printer was used to apply 8 coats of matrix at a flow rate of $0.1 \mathrm{~mL} /$ minute at $40{ }^{\circ} \mathrm{C}$ with a gas pressure of $50 \mathrm{psi}$ and a $z$ height of $30 \mathrm{~mm}$. The velocity was set within the G-code as F1000, which resulted in a velocity of $1100 \mathrm{~mm} /$ minute. Matrix area density was $0.11 \mathrm{mg} / \mathrm{cm}^{2}$.

Matrix application of mouse brain: The matrix solution was $10 \mathrm{mg} / \mathrm{mL}$ of $\alpha$-cyano-4-hydroxycinnamic acid (CHCA) in $60: 40(\mathrm{v} / \mathrm{v})$ acetonitrile: water with $0.1 \%$ TFA. The velocity was set within the G-code as F800, which resulted in a veloci- ty of $800 \mathrm{~mm} /$ minute. The total time taken for 4 passes of a glass slide was 20 minutes. For consistent performance, regular wash cycles were done with solvent before and after use. Matrix area density was $0.22 \mathrm{mg} / \mathrm{cm}^{2}$.

Mass spectrometry imaging. A $12 \mathrm{~T}$ SolariX FT-ICR MS (Bruker Daltonics, Bremen, Germany) equipped with a MALDI ionization source was operated in negative ionization mode to acquire spectra between $98-1500 \mathrm{~m} / \mathrm{z}$. The laser raster increment was set to $40 \mu \mathrm{m}$ along both the $x$ and $y$ axis with a smart walk of $50 \mu \mathrm{m}$. Laser focus was set to minimum, with a frequency of $1000 \mathrm{~Hz}$. Ions were accumulated across 300 laser shots for each mass analysis. For mouse brain analysis, source was operated in positive ionization mode to acquire spectra between $250-2500 \mathrm{~m} / \mathrm{z}$. The laser raster increment was set to $75 \mu \mathrm{m}$ along both the $x$ and $y$ axis with a smart walk of $50 \mu \mathrm{m}$. Laser focus was set to small, with a frequency of $1000 \mathrm{~Hz}$. Ions were accumulated across 300 laser shots for each mass analysis. Calibration of the spheroid data was performed postprocessing using a pre-determined list of internal calibrants (supplementary information table 1). The images were then analyzed without normalization using FlexImaging 4.1 (Bruker Daltonics, Bremen, Germany) and discriminatory analysis completed using SCiLS (Bruker Daltonics, Bremen, Germany). The metabolites were identified using the Human Metabolome Database (HMDB) using an error tolerance of 10 ppm for $[\mathrm{M}-\mathrm{H}]^{-}$. (http://www.hmdb.ca/spectra/ms/search).

H\&E Staining. Sectioned spheroids were placed onto a glass slide and stored in a vacuum desiccator (RT, 18h) Cells were first washed with water. Sections were covered with haematoxylin (Sigma-Aldrich, Missouri, USA) (3 minutes) and washed with running water. The slide was coated with tap water (1 minute), then acid alcohol (Sigma-Aldrich, Missouri, USA) (20 seconds) and then washed using water. Eosin (Sigma-Aldrich, Missouri, USA) was added (1 minute) and then washed with water. The stained cells were then air dried before being imaged under the 40X objective on an inverted AE2000 (Motic, Hong Kong) microscope.

\section{Results and Discussion}

Optimization of MALDI matrix application using rhodamine B microarrays. Delocalization during matrix application was measured using microarrays of rhodamine $\mathrm{B}$ spots $(0.01 \% \mathrm{w} / \mathrm{v})$ printed on glass slides. Rhodamine B is a watersoluble fluorescent dye which provided a model of low molecular weight metabolites that commonly delocalize in MALDI MSI. 45 individual spots per slide provided a high number of technical repeats to produce statistically robust data for assessing the extent of delocalization. Each slide was coated with a MALDI matrix (9AA) using the 3D-printer platform and the average change in area was calculated. To identify the optimal matrix application conditions for minimizing delocalization, a user defined experimental design was compiled using Design Expert 10 (Stat-Ease, Minneapolis, USA) with discrete increments of 10 for 3 matrix application variables: nebulizing gas pressure $(40-60 \mathrm{psi})$, build-plate temperature $\left(20-40{ }^{\circ} \mathrm{C}\right)$ and nebulizer $z$ height (i.e. height of nebulizer above the sample surface) $(10-30 \mathrm{~mm})$. No voltage was applied as the nebulizer was used for nebulization. This process produced a set of 27 discrete conditions for matrix application. A slide of 45 replicate rhodamine B spots was coated using each of the 27 defined conditions. The change in area of fluorescence before 
and after matrix application was recorded using a BioAnalyzer 4F/4S (LaVision BioTec, Bielefeld, Germany) fluorescence scanner and measured using ImageJ, In order to quantitate analyte diffusion as a consequence of matrix application, the average area change in fluorescence area after matrix coating was calculated (figure 3 and supplementary information table 2).

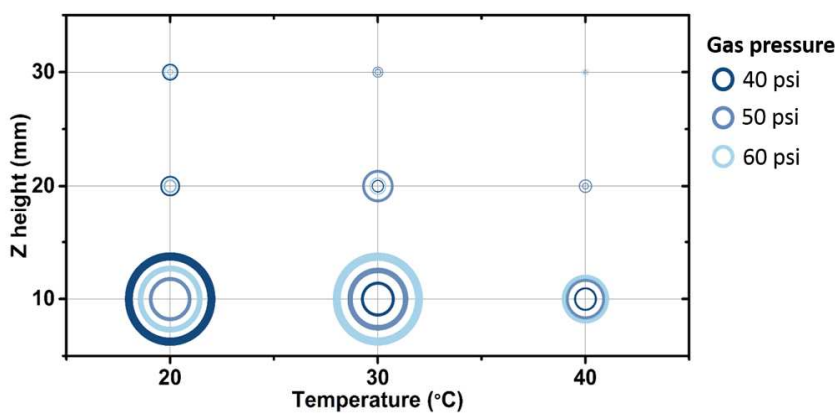

Figure 3. A bubble plot showing how the 27 matrix application conditions affect the change in area of rhodamine B fluorescence. The size of the circle is the percentage change in area of fluorescence after matrix application (diffusion) and the dark to pale blue color scale represents increasing gas pressure.

By comparing the data collected over all the conditions tested, it can be seen that increasing both the $z$ height and temperature reduced the extent of rhodamine B delocalization (supplementary figure 2). In contrast, varying the gas pressure had a lesser effect on the delocalization. The optimized parameters of 40 ${ }^{\circ} \mathrm{C}$ build-plate temperature, $30 \mathrm{~mm}$ nebulizer $z$ height and 50 psi gas pressure produced a dry matrix application with a 9.44 $\%$ change in fluorescence area after matrix application. In contrast, the worst conditions were identified as $20{ }^{\circ} \mathrm{C}, 10 \mathrm{~mm}$ $z$ height and 60 psi gas pressure, producing a $403 \%$ increase in area of rhodamine B with visible smearing of the rhodamine B spots after matrix application (figure 4).

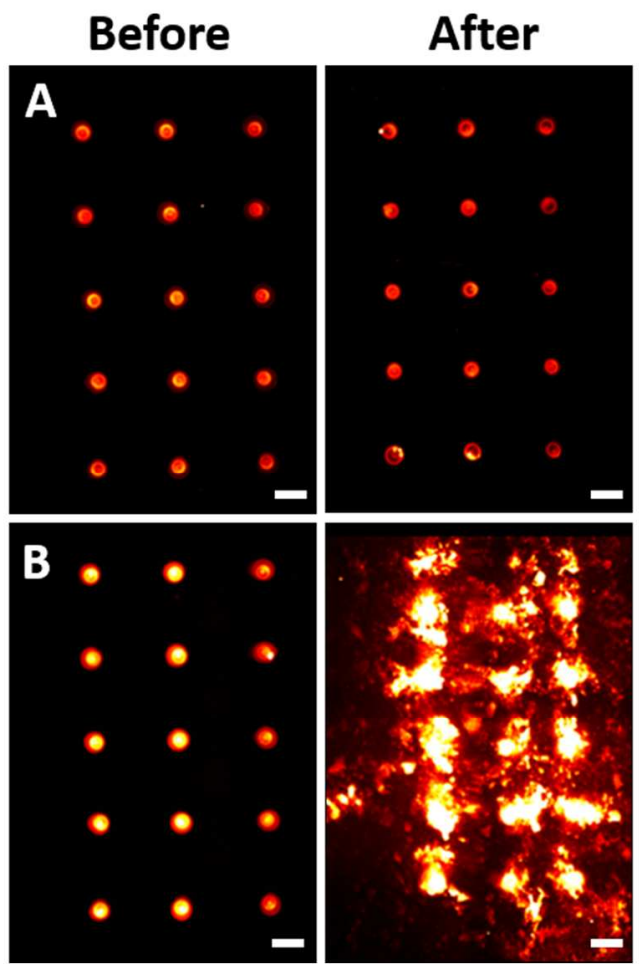

Figure 4. Rhodamine B spots $(0.01 \% \mathrm{w} / \mathrm{v}, 17.5 \mu \mathrm{L})$ were spotted using a microarray printer, and the fluorescence imaged at $70 \mathrm{~ms}$ exposure. The change in area was measured for all 27 conditions tested. Here the images of the optimized (A, $\left.40{ }^{\circ} \mathrm{C}, 30 \mathrm{~mm}, 50 \mathrm{psi}\right)$ and worst $\left(\mathrm{B}, 20{ }^{\circ} \mathrm{C}, 10 \mathrm{~mm}, 60 \mathrm{psi}\right)$ conditions before and after matrix application are shown. Scale bar $1 \mathrm{~mm}$.

Imaging the MALDI matrix crystal size using scanning electron microscopy. The crystal size produced during matrix application defines the spatial resolution and the extent of analyte diffusion. ${ }^{11}$ By reducing the crystal size, a greater spatial resolution is achieved. ${ }^{9}$ To assess the size of the matrix crystals, scanning electron microscopy (SEM) was used. Crystal size on the rhodamine printed slides was determined for both the optimized and worst parameters for the modified 3D printer platform, as well as for our laboratory's standard application protocol using a commercially available system (the HTX TM-sprayer) (figure 5). SEM images revealed that the worst conditions produced a coverage of large heterogeneous crystals. In contrast, the optimized conditions produced a homogenous coating of small regularly-sized crystals across the sample which were comparable to that of the commercial printer.
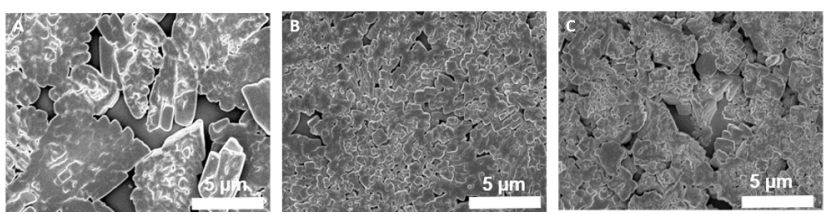

Figure 5. Scanning electron microscope images of the crystals produced under the $(\mathrm{A})$ worst $3 \mathrm{D}$ printer conditions $(\mathrm{B})$ optimized 3D printer conditions and (C) optimized commercial HTX TM-sprayer conditions. The worst conditions produced large inhomogeneous crystals whilst the optimized method 
produced small crystals with a uniform coverage that were similar to those observed using the commercial sprayer.

MALDI imaging of metabolites in tissue. Multicellular tumor spheroids (MTS) are spherical self-assembled cell cultures that grow up to $1 \mathrm{~mm}$ in diameter and are the most commonly used 3D model for assessing drug penetration and efficacy ex vivo. ${ }^{23,24}$ MTS are known to have a diffusion limit of $150-200 \mu \mathrm{m}$ to molecules including oxygen, glucose and other metabolites, above which the inefficient mass transport causes metabolic waste accumulation and gradients of nutrients and waste products. ${ }^{25,26}$ A number of groups have used MSI to observe the distribution of proteins ${ }^{27}$ and drugs ${ }^{28}$ within spheroids. However due to their small size any delocalization caused by matrix application can greatly affect the biological validity of the results obtained. Based on the optimal conditions obtained through experimental design, MCF-7 spheroids were sectioned and subsequently imaged using MALDI FT-ICR MS at $40 \mu \mathrm{m}$ imaging resolution. These sub-1 $\mathrm{mm}$ tissues provide an effective model to understand the spatial distribution of molecules in small systems. The resulting MSI data achieved using the optimized and non-optimal 3D printer matrix application conditions were compared to the current laboratory standard protocol using a HTX-TM sprayer. Figure 6 shows the distribution of a number of metabolites including glutathione, adenosine triphosphate (ATP) and adenosine monophosphate (AMP) throughout the spheroid beside an optical image of an adjoining H\&E stained section to validate the spheroid morphology after cryosectioning. Without optimization, the home-built matrix sprayer produced extensive diffusion of molecules including glutathione. This resulted in the analytes diffusing out of the tissue and into the embedding medium, producing a biologically inaccurate distribution. The nebulizer was located near the sample at a high gas pressure without a heated bed. Under these lower temperature conditions, crystals form more slowly and therefore produce larger heterogeneous distributions, incorporating analytes across larger regions with a poor signal-to-noise ratio for the analytes observed. ${ }^{9}$ However, through optimization of the home-built matrix sprayer, including increasing the $z$ height of the nebulizer and increasing the temperature of the heated bed, the distributions resembled those observed under the optimized conditions of a commercially available sprayer, with a number of analytes having an improved signal from that observed using the commercial sprayer. Glutathione was observed to entirely delocalize outside of the spheroid prior to the home-built matrix sprayer optimization, however under the optimized conditions it localized towards the centre of the spheroid, similarly to that observed using the commercially available sprayer. A similar distribution was observed for the oxidized form of glutathione. In contrast, ATP distributed to the outer region of the spheroid under all spraying conditions. The most noticeable difference as a result of the optimization is the distribution of AMP. After optimization, AMP appears uniformly distributed, suggesting that the localization to the outer part of the spheroid that is observed using non-optimized matrix deposition is an artefact caused by analyte delocalization. Importantly, these distributions were reproducibly observed using both the commercially available sprayer and the 3D printer (supplementary figure

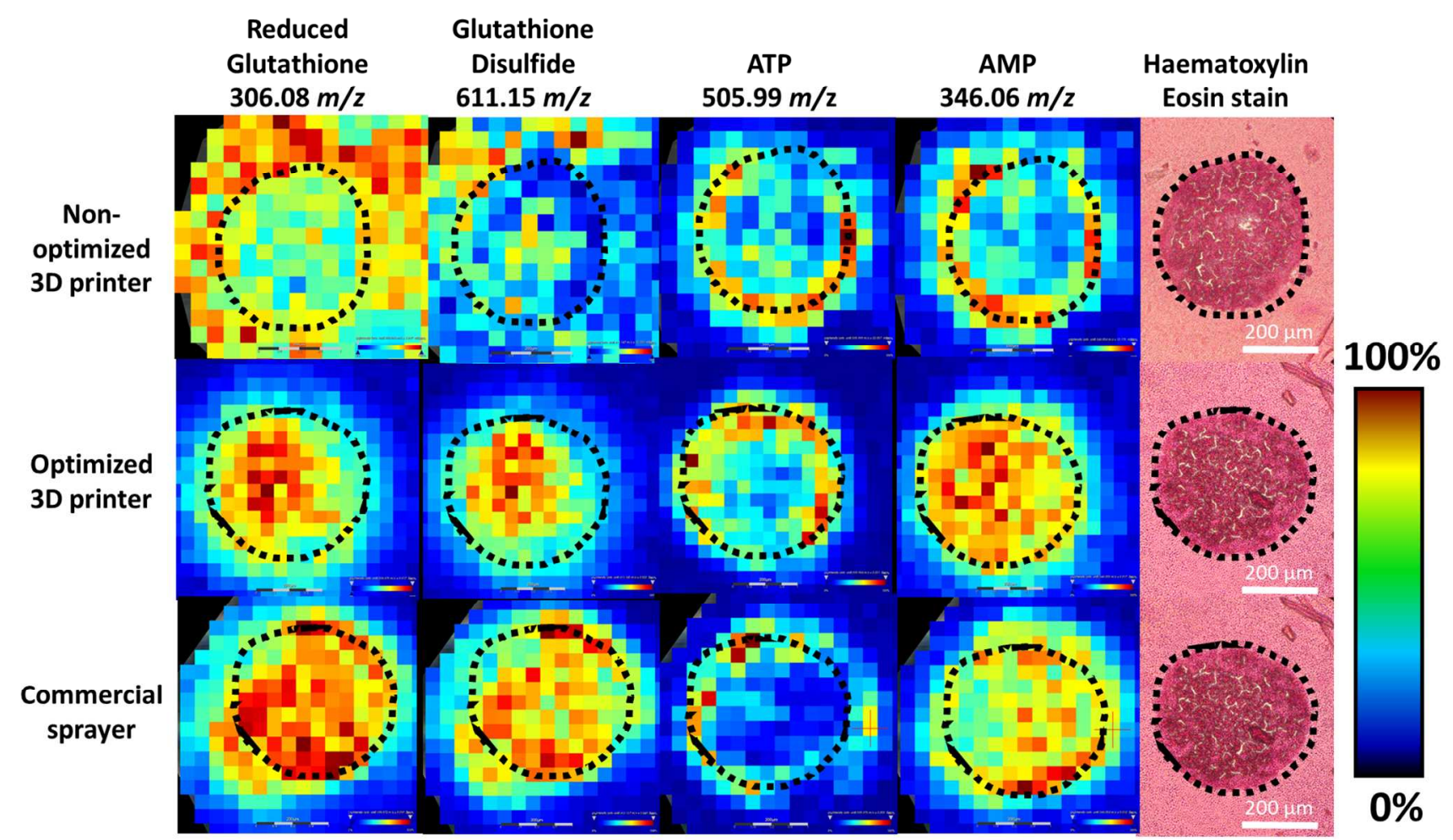

Figure 6. MCF-7 spheroids were imaged at $40 \mu \mathrm{m}$ lateral laser resolution using the FT-ICR MS. Distributions of molecules were observed and compared between the non-optimized and optimized parameters of the 3D printer compared to that of the current protocol using a commercially available sprayer. The H\&E sections are shown to confirm the morphology of the cryosectioned spheroids. 
To further demonstrate the reproducibility and efficacy of the matrix coater over a larger area and with alternative matrices, the $3 \mathrm{D}$ printer was used to coat sections of mouse brain with CHCA. The sample was imaged at $75 \mu \mathrm{m}$ lateral laser resolution using FT-ICR MS (figure 7). The spatial distributions of two endogenous steroids, derivatized with Girard's reagent T (as described previously (22)) were determined (figure 7B and
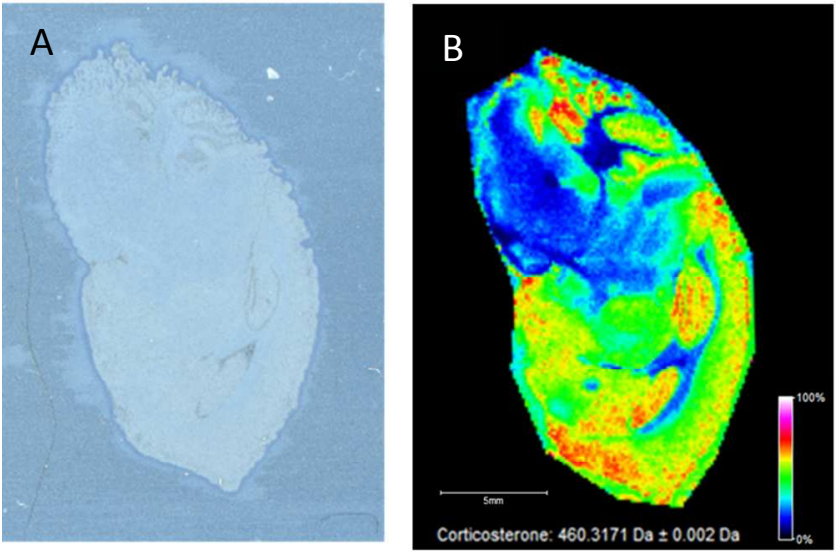

Fig 7C). The observed distributions closely matched previously reported results. ${ }^{22}$ Importantly, the spatial distribution of the data was consistent throughout the $\sim 20 \mathrm{~mm}$ tissue section, with clear partitioning of the endogenous steroids throughout the tissue. These observations confirm the consistency of matrix deposition using our matrix application platform.

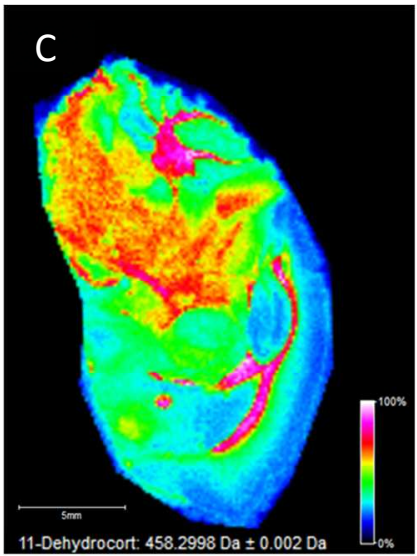

Figure 7. The 3D printer was used to coat sections of mouse brain with CHCA as the matrix. The spatial distributions of two endogenous steroids were observed and found to closely match previously reported results. ${ }^{22}$ Tissue was imaged at $75 \mu \mathrm{m}$ lateral laser resolution using the FT-ICR MS. (A) optical image of sagittal cryosection of murine brain, (B) MSI heat map of Corticosterone derivative (GirT-CORT) at m/z $460.3171 \pm 0.002 \mathrm{Da},(\mathrm{C}) 11$-dehydrocorticosterone (GirT-11DHC) at $\mathrm{m} / \mathrm{z} 458.2998 \pm 0.002 \mathrm{Da}$ and (D) MSI images superimposition of corresponding steroids derivatives. Signal intensity is depicted by colour on the scale shown. Scale bar $(5 \mathrm{~mm})$.

\section{Conclusion}

Here we have shown that a 3D printer can be converted into an effective automated MALDI matrix applicator which operates with minimal analyte diffusion during matrix application. Using fluorescent microarrays and experimental design the conditions for 9AA matrix application were optimized. With a heated bed and an increased $z$ height of the sprayer, it was possible to minimize sample wetting and therefore reduce analyte diffusion. These optimized conditions could then be used to reproducibly image MCF-7 spheroids and identify numerous metabolites with reduced analyte delocalization. This produced images with comparable distributions to those observed using a commercially available printer at a fraction of the set-up cost compared to commercially available systems. The efficacy of the device was further demonstrated over larger areas and with alternative matrices by imaging a mouse brain coated with CHCA. As a result, initial findings shown here demonstrate the potential application of this system for effective MALDI matrix application to biological tissue sections, thus enabling reliable low-cost high-resolution MSI.

\section{ASSOCIATED CONTENT}

\section{Supporting Information}

The supporting information is available free of charge on the ACS publications website.

A schematic of the 6-port valve used for MALDI-matrix deposition (PDF)

Experimental design using a user defined method with discrete increments of 10 for 3 parameters of the converted 3D printer: temperature, $z$ height and gas pressure (PDF)

Calibration list used for MALDI-MSI of MCF-7 spheroids (PDF)
The file to print the 3D block used to convert the printer (.STL) Detailed information regarding conversion of the $3 \mathrm{D}$ printer and the raw imaging files of the MCF-7 spheroids using the different printing conditions (imzML) have been deposited on Edinburgh DataShare (https://datashare.is.ed.ac.uk/handle/10283/3014).

\section{AUTHOR INFORMATION}

\section{Corresponding Author}

*Email:1mackay@ed.ac.uk. Phone: 01316513048

\section{Author Contributions}

C.L.M and D.J.C. conceived the project. C.L.M provided guidance in re-building the printer. L.H.T. collected the data and wrote the manuscript. A.C-G. provided the training and assisted in microarray printing. G.R.H., C.L.M., C.J.C., D.J.C. R.J.A.G., and D.C. all contributed guidance on the manuscript. All authors have given approval to the final version of the manuscript.

\section{ACKNOWLEDGMENT}

The authors wish to acknowledge the assistance of Steve Mitchell with SEM imaging in the School of Biological Sciences, University of Edinburgh. Funding was provided by AstraZeneca and an EPSRC CASE-studentship to L. H. T.

\section{REFERENCES}

(1) Swales, J. G.; Tucker, J. W.; Strittmatter, N.; Nilsson, A.; Cobice, D.; Clench, M. R.; Mackay, C. L.; ; Andren, P.E.; Takáts, Z.; Webborn, P. J. H.; Goodwin, R. J. A. Anal. Chem. 2014, 86 (16), 8473-8480.

Hart, P. J.; Francese, S.; Claude, E.; Woodroofe, M. N.; Clench, M. R. Anal. Bioanal. Chem. 2011, 401 (1), 115-125.

(3) Chaurand, P.; Caprioli, R. M. Electrophoresis 2002, 23 (18), $3125-3135$ 
(4) Chaurand, P.; Schwartz, S. A.; Caprioli, R. M. Anal. Chem. 2004, 76(5), 86A - 93A.

(5) Dill, A. L.; Eberlin, L. S.; Costa, A. B.; Ifa, D. R.; Cooks, R. G. Anal. Bioanal. Chem. 2011, 401 (6), 1949-1961.

(6) Chughtai, K.; Heeren, R. M. A. Chem. Rev. 2010, 110 (5), 3237-3277.

(7) Dill, A. L.; Eberlin, L. S.; Ifa, D. R.; Cooks, R. G. Chem. Comms 2011, 2741-2746.

(8) Feenstra, A. D.; Dueñas, M. E.; Lee, Y. J. J. Am. Soc. Mass. Spectrom. 2017, 434-442.

(9) Goodwin, R. J. A. J. Proteomics 2012, 75 (16), 4893-4911.

(10) Heeren, R. M. A.; Kükrer-Kaletas, B.; Taban, I. M.; MacAleese, L.; McDonnell, L. A. Appl. Surf. Sci. 2008, 255 (4), 1289-1297.

(11) Schwartz, S. A.; Reyzer, M. L.; Caprioli, R. M. J. Mass Spectrom. 2003, 38 (7), 699-708.

(12) Gemperline, E.; Rawson, S.; Li, L. Anal. Chem. 2014, 86 (20), 10030-10035.

(13) Aerni, H. R.; Cornett, D. S.; Caprioli, R. M. Anal. Chem. 2006, $78(3), 827-834$.

(14) Eriksen, J.; Jørgensen, T. N.; Gether, U. J. Neurochem. 2010, $113(1), 27-41$.

(15) Lloro, I.; Bueno, A.; Calvo, J.; Urreta, H.; Elortza, F. J. of Lab Automation 2016, 21 (2), 260-267

(16) Baluya, D. L.; Garrett, T. J.; Yost, R. A. Anal. Chem. 2007, 79 (17), 6862-6867.

(17) Soleimani-Gorgani, A. Inkjet Printing; Elsevier Inc., 2015, 231-
246.

(18) Baluya, D. L.; Garrett, T. J.; Yost, R. A. 2007, 79 (17), 68626867.

(19) Zarrin, F.; Kaufman, S. L.; Socha, J. R. J. Aerosol Sci. 1991, 22 (SUPPL. 1), 343-346.

(20) Reschke, B. R.; Timperman, A. T. J. Am. Soc. Mass Spectrom. 2011, 22 (12), 2115-2124.

(21) Jamieson, L.E.; Camus, V.L.; Bagnaninchi, P.O.; Fisher, K.M.; Stewart, G. D.; Nailon, W. H.; Mclaren, D. B.; Harrison, J.; Campbell, C. J. Analyst 2016, 16710-16718.

(22) Cobice, D.F.; Mackay, C.L.; Goodwin, R.A.; McBride, A.; Langridge-Smith, P.R.; Webster, S.P.; Walker, B.R.; Andrew, R.A. Anal. Chem. 2013, 85 (23), 11576-11584.

(23) Mehta, G.; Hsiao, A. Y.; Ingram, M.; Luker, G. D.; Takayama, S. J. Control. Release 2012, 164 (2), 192-204.

(24) Jamieson, L. E.; Harrison, D. J.; Campbell, C. J. Analyst 2015 $86(20), 10030-10035$.

(25) Alvarez-Pérez, J.; Ballesteros, P.; Cerdán, S. Magn. Reson. Mater. Physics, Biol. Med. 2005, 18 (6), 293-301.

(26) Hirschhaeuser, F.; Menne, H.; Dittfeld, C.; West, J.; MuellerKlieser, W.; Kunz-Schughart, L. A. J. Biotechnol. 2010, 148 (1), $3-15$.

(27) Li, H..; Hummon, A. Anal Chem 2011, 83 (22), 8794-8801.

(28) Liu, X.; Weaver, E. M.; Hummon, A. B. Anal. Chem. 2013, 85 (13), 6295-6302. 
for TOC only

3

4

5

6

7

8

9

10

11

12

13

14

15

16

17

18

19

20

21

22

23

24

25

26

27

28

29

30

31

32

33

34

35

36

37

38

39

40

41

42

43

44

45

46

47

48

49

50

51

52

53

54

55

56

57

58

59

60 